\title{
COMITÉ CIENTÍFICO
}

PhD. Diego Andrés Guevara Fletcher Comisión de Regulación de Telecomunicaciones Argentina

PhD. Ing. Darío Amaya Hurtado

UNIVERSIDAD DISTRITAL FRANCISCO JOSÉ DE CALDAS

Facultad De Ingeniería

\section{DIRECTIVOS DE LA FACULTAD}

\section{RECTOR}

Dr. Inocencio Bahamon Calderón

\section{DECANO}

Msc. Ing. Octavio José Salcedo Parra.

\section{REVISTA REDES DE INGENIERÍA}

\section{DIRECTOR:}

Msc. Ing. Danilo Alfonso López Sarmiento. Universidad Distrital "Francisco José de Caldas". Bogotá, Colombia.

\section{EDITOR:}

Msc. Ing. Danilo Alfonso López Sarmiento. Universidad Distrital "Francisco José de Caldas". Bogotá, Colombia.

\section{COMITÉ EDITORIAL}

PhD. Ing. Jaime Alberto Guzmán Luna Universidad Nacional de Colombia Bogotá, Colombia

PhD. Ing. Gustavo López Rubio Universidad Pontificia de Salamanca España

$\mathrm{PhD}$. Ing. Luis Humberto Sarmiento Suescum Universidad Manuela Beltrán Bogotá, Colombia

PhD. Ing. Oscar Gualdrón González Universidad de Pamplona Norte de Santander, Colombia

MSc. Ing. Luis Fernando Martínez Pedraza Universidad Distrital "Francisco José de Caldas" Bogotá, Colombia

Universidad Militar Nueva Granada

Bogotá, Colombia

PhD (c). Ing. José Félix Vega Stavro

Universidad Nacional

Bogotá, Colombia

MSc. Ing. Octavio José Salcedo Parra

Universidad Distrital "Francisco José de Caldas"

Bogotá, Colombia

MSc. Ing. Cesar Augusto Hernández Suarez

Universidad Distrital "Francisco José de Caldas" Bogotá, Colombia

\section{COMITÉ EVALUADOR}

PhD (c). Ing. Fredy Hernán Martínez Sarmiento. Universidad Distrital "Francisco José de Caldas" Bogotá, Colombia

MSc. Luis Fernando Martínez Pedraza

Universidad Distrital "Francisco José de Caldas" Bogotá, Colombia

PhD. Ing. Héctor Fernando Cancino de Greiff Universidad Distrital "Francisco José de Caldas" Bogotá, Colombia

MSc. Ing. Luis Efrén Barrera

Universidad de los Andes

Bogotá, Colombia

MSc. Ing. Juan Manuel Sánchez Céspedes

Universidad Manuela Beltrán

Bogotá, Colombia

MSc. Ing. Danilo Alfonso López Sarmiento Universidad Distrital "Francisco José de Caldas" Bogotá, Colombia

MSc. Ing. Pablo German Perez Universidad Militar Nueva Granada Bogotá, Colombia 


\section{ASISTENTES DE LA REVISTA}

Tlgo. Diego Armando Giral Ramírez

\section{PORTADA}

Nombre: Pozos Azules Villa de Leyva - Colombia Fotografía: Cindy Estefany Guerrero Cifuentes

\section{EDITORIAL}

Universidad Distrital "Francisco José de Caldas" Miembro asociación de editorial Universitarias de Colombia

\section{CORRECCIÓN DE ESTILO}

MSc. Ing. Cesar Augusto Hernández Suarez.

\section{CORRECCIÓN DE ESTILO PARA INGLES}

Ing. Felipe Forero

\section{DISEÑO DE CUBIERTA}

Tlgo. Diego Armando Giral Ramírez

\section{DIAGRAMADOR}

Tlgo. Diego Armando Giral Ramírez

\section{REVISTA REDES DE INGENIERÍA}

Redes de Ingeniería es una revista electrónica Institucional adscrita a la Facultad de Ingeniería de la Universidad Distrital "Francisco José de Caldas".

Es una revista de carácter semestral que publica los resultados de Investigación, revisión, reflexión de la comunidad académico-científica los meses Junio y Diciembre de cada año. Posee un sistema de divulgación netamente electrónico y su primer número fue publicado en Junio del 2010.

Redes de Ingeniería es una revista arbitrada mediante un proceso de revisión entre pares de doble ciego. Las opiniones expresadas de esta publicación pueden ser reproducidas citando la fuente.

\section{COBERTURA TEMÁTICA}

Las líneas de publicación de la revista están enmarcadas en las áreas de las Telecomunicaciones, Electróni- ca, Sistemas, Bioingeniería, Eléctrica, Medio Ambiente, Educación, Industrial, Geomática; además de todos aquellos temas que converjan hacía la Ingeniería.

\section{MISIÓN}

La revista Redes de Ingeniería tiene el objetivo de servir como medio de divulgación de los resultados obtenidos a través procesos de investigación, reflexión, indagación en entornos Educativos y Empresariales en temas de actualidad en "pro" del mejoramiento de la calidad de vida de la sociedad en general.

\section{PÚBLICO OBJETIVO}

Investigadores, docentes, estudiantes y demás profesionales de las ramas de la Ingeniería que deseen estar a la vanguardia de las teorías y/o tecnologías que rigen y regirán el mundo moderno desde un enfoque científico.

\section{INDEXACIÓN}

La revista Redes de Ingeniería es una publicación electrónica académica indexada en el Índice Bibliográfico Nacional Publindex (IBN) de Colciencias (Colombia) en categoría C desde el 1 de Enero de 2012.

\section{SUSCRIPCIÓN}

No tiene ningún costo y para la realización del registro utilice el formato que aparece ubicado al final de la revista y por favor envíelo al correo o diríjase directamente a la Web.

\section{REPRODUCCIÓN}

Los textos de los artículos son publicados bajo la responsabilidad de los Autores y no reflejan el pensamiento de la revista, ni comprometen de ninguna manera a la Universidad Distrital "Francisco José de Caldas".

\section{DIRECCIÓN}

Universidad Distrital "Francisco José de Caldas" Facultad de Ingeniería (Carrera 7 \# 40 -53, Piso cuarto) Especialización en Teleinformática. Bogotá, Colombia (Sur América)

redesdeingenieria@udistrital.edu.co redesdeingenieria@gmail.com

http://ingenierial.udistrital.edu.co/digital/index.php/ redesdeingeneiria/index

\section{R (DES DE INGENIERÍA

